

S. Chobanyan, H. Salehi, Exact maximal inequalities for exchangeable systems of random variables, Теория вероятн. и ее примен., 2000, том 45, выпуск 3, 555-567

DOI: https://doi.org/10.4213/tvp485

Использование Общероссийского математического портала Math-Net.Ru подразумевает, что вы прочитали и согласны с пользовательским соглашением http://www.mathnet.ru/rus/agreement

Параметры загрузки:

IP: 3.93 .64 .190

26 апреля 2023 г., 14:09:05

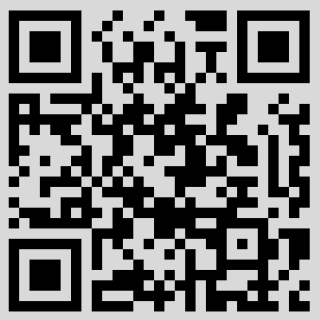




\title{
EXACT MAXIMAL INEQUALITIES FOR EXCHANGEABLE SYSTEMS OF RANDOM VARIABLES ${ }^{1)}$
}

\begin{abstract}
Для конечной системы $\left(\xi_{1}, \ldots, \xi_{n}\right)$ перестановочных случайных величин со значениями в банаховом пространстве таких, что $\sum_{1}^{n} \xi_{i}=0$, доказывается эквивалентность в смысле (2) величин $\mathbf{E} \Phi\left(\max _{k \leqslant n}\left\|\xi_{1}+\cdots+\xi_{k}\right\|\right)$ и $\mathbf{E} \Phi\left(\left\|\sum_{1}^{n} \xi_{i} r_{i}\right\|\right)$ для любой возрастающей выпуклой функции $\Phi: \mathbf{R}^{+} \rightarrow \mathbf{R}^{+}, \Phi(0)=0$, где $\left(r_{1}, \ldots, r_{n}\right)-$ система случайных величин Радемахера, не зависящих от $\left(\xi_{1}, \ldots, \xi_{n}\right)$. Устанавливается также эквивалентность хвостов соответствующих распределений. Эти результаты, по-видимому, являются новыми и для скалярных случайных величин. В качестве следствия найдены наилучшие оценки среднего для $\max _{k \leqslant n}\left\|a_{\pi(1)}+\cdots+a_{\pi(k)}\right\|$ относительно всех перестановок $\pi$ неслучайных векторов $a_{1}, \ldots, a_{n}$ из нормированного пространства.
\end{abstract}

Ключевые слова и фразы: перестановочные случайные величины, банахово пространство, максимальное неравенство, перестановки.

Introduction. The aim of this paper is to give various versions of maximal inequalities for finite systems of exchangeable random variables, scalar or with values in a Banach space. It is well known that infinite exchangeable systems of random variables enjoy nice properties due to the fact that they are conditionally independent with respect to the tail $\sigma$-algebra. This fact fails to be true for general finite systems.

In 1974 Maurey and Pisier have shown that for exchangeable systems of random variables $\xi_{1}, \ldots, \xi_{n}$ with $\sum_{1}^{n} \xi_{j}=0$ the value $\mathbf{E} \max _{k \leqslant n} \| \xi_{1}+$ $\cdots+\xi_{k} \|^{p}$ is equivalent (can be estimated both from below and above) to $\mathbf{E}\left\|\sum_{1}^{n} \xi_{j} r_{j}\right\|^{p}$, where $r_{1}, \ldots, r_{n}$ are Rademacher random variables independent of $\left(\xi_{1}, \ldots, \xi_{n}\right)$.

It is interesting to note that some consequences of the result for a long time have not been noticed by analysts. One such consequence is the gener-

\footnotetext{
${ }^{*}$ Институт вычислительной математики им. Н.И. Мусхелишвили АН Грузи,, ул. Акурп, 8, 380093 Тбилиси, Грузия; e-mail: chob@scien.compmath.acnet.ge

${ }^{* *}$ Michigan State University, East Lansing, MI 48824, USA; e-mail: salehi@stt.msu.edu

1) The first author was partly supported by the Georgian Academy of Sciences, Grant 1.16.
} 
alization of the Garsia result estimating the expression

$$
\frac{1}{n !} \sum_{\pi} \max _{k \leqslant n}\left|\sum_{1}^{k} a_{\pi(i)}\right|^{p}, \quad 1 \leqslant p<\infty,
$$

for given numbers $a_{1}, \ldots, a_{n}$ with $\sum_{1}^{n} a_{j}=0$, where $\pi$ runs over all possible permutations of $\{1, \ldots, n\}$. A consequence of Maurey and Pisier result permits to establish exact estimation of $(*)$ for vectors $a_{1}, \ldots, a_{n}$ of a $\mathrm{Ba}$ nach space playing a crucial role in the theory of rearranged functional series (see [5], [2], [9]).

In Section 1 we show that the Maurey and Pisier result can be generalized to the case of general increasing and convex functions $\Phi$. Our presentation uses a special trick (see Lemma) that allows one to get the desired inequalities immediately in an elementary way.

Section 2 deals with applications. Besides generalizations of the Garsia result we point out the following curious fact. Let $\xi_{1}, \ldots, \xi_{n}$ be i.i.d. random variables with a continuous distribution and let $\zeta_{i}=\left(\xi_{i}-\bar{\xi}\right) / s, i=1, \ldots, n$, where $\bar{\xi}$ is the sample mean, $\bar{\xi}=\left(\xi_{1}+\cdots+\xi_{n}\right) / n$, while $s$ is the sample standard deviation, $s^{2}=\sum_{1}^{n}\left(\xi_{j}-\bar{\xi}\right)^{2} / n$. Then for each $p, 1 \leqslant p<\infty$, there are absolute constants $c_{p}>0$ and $C_{p}>0$ such that $c_{p} n^{p / 2} \leqslant \mathbf{E} \max _{k \leqslant n} \mid \zeta_{1}+$ $\cdots+\left.\zeta_{k}\right|^{p} \leqslant C_{p} n^{p / 2}$. The result remains valid for exchangeable systems in a Banach space after specifying a proper analog of $s$.

Section 3 contains the main result of the paper. Here we find bilateral inequalities for $\mathbf{P}\left\{\max _{k \leqslant n}\left\|\xi_{1}+\cdots+\xi_{k}\right\|>t\right\}$ in the normed space case.

In Section 4 there are given moment maximal inequalities with multipliers. We find upper estimations for $\mathbf{E} \max _{k \leqslant n}\left\|A_{1} \xi_{1}+\cdots+A_{k} \xi_{k}\right\|^{p}, 1 \leqslant p<\infty$, where $A_{1}, \ldots, A_{k}$ are linear bounded operators in the Banach space, where the random variables of an exchangeable system take their values. We follow the line suggested in [8] reducing the problem to the case of identity operators, $A_{1}=\cdots=A_{n}=I$, that is considered in Section 1.

1. Moment maximal inequality. Let $X$ be a real or complex Banach space. By an $X$-valued random variable we mean a Bochner measurable mapping $\xi: \Omega \rightarrow X$ defined on underlying probability space $(\Omega, \mathscr{A}, \mathbf{P})$. A finite system $\xi_{1}, \ldots, \xi_{n}$ of $X$-valued random variables is called exchangeable, if for any permutation $\pi$ of $\{1, \ldots, n\}$ the joint distribution of $\left(\xi_{\pi(1)}, \ldots, \xi_{\pi(n)}\right)$ coincides with that of $\left(\xi_{1}, \ldots, \xi_{n}\right)$. By a (finite or countable) family $\left(r_{i}\right)$ of Rademacher random variables we mean independent random variables taking values \pm 1 with probabilities $\frac{1}{2}$.

The aim of this section is to prove the following

Theorem 1.1. Let $\left(\xi_{1}, \ldots, \xi_{n}\right)$ be a system of exchangeable $X$-valued random variables with $\sum_{1}^{n} \xi_{j}=0$, and let $\Phi: \mathbf{R}^{+} \rightarrow \mathbf{R}^{+}, \boldsymbol{\Phi}(0)=0$, be any continuous increasing convex function. Then 
a) For any collection $\left(\theta_{1}, \ldots, \theta_{n}\right)$ of signs $\theta_{j}= \pm 1$

$$
\mathbf{E} \Phi\left(\max _{k \leqslant n}\left\|\sum_{1}^{k} \xi_{j}\right\|\right) \leqslant \mathbf{E} \Phi\left(\max _{k \leqslant n}\left\|\sum_{1}^{k} \xi_{j} \theta_{j}\right\|\right)
$$

b) The following two-sided inequality holds

$$
\mathbf{E} \Phi\left(\frac{1}{2}\left\|\sum_{1}^{n} \xi_{j} r_{j}\right\|\right) \leqslant \mathbf{E} \Phi\left(\max _{k \leqslant n}\left\|\sum_{1}^{k} \xi_{j}\right\|\right) \leqslant 2 \mathbf{E \Phi}\left(\left\|\sum_{1}^{n} \xi_{j} r_{j}\right\|\right),
$$

where $\left(r_{j}\right)$ is a family of Rademacher random variables which is independent of $\left(\xi_{1}, \ldots, \xi_{n}\right)$.

An elementary lemma below plays a crucial role in the proof of the theorem. Let $\left(b_{1}, \ldots, b_{n}\right)$ be a collection of elements of $X$. Denote

$$
|b|=\left|\left(b_{1}, \ldots, b_{n}\right)\right|=\max _{k \leqslant n}\left\|b_{1}+\cdots+b_{k}\right\|
$$

$|\cdot|$ represents a norm in the space of ordered collections $\left(b_{1}, \ldots, b_{n}\right)$.

Lemma. If $\sum_{1}^{n} b_{j}=0$, then

a) For each $k, 1 \leqslant k \leqslant n$,

$$
\max \left(\left|\left(b_{1}, \ldots, b_{k}\right)\right|,\left|\left(b_{k+1}, \ldots, b_{n}\right)\right|\right)=\left|\left(b_{1}, \ldots, b_{k}, b_{n}, b_{n-1}, \ldots, b_{k+1}\right)\right|
$$

b) For any collection of signs $\theta=\left(\theta_{1}, \ldots, \theta_{n}\right)$ there exists a permutation $\pi(\theta)$ of $\{1, \ldots, n\}$ such that

$$
|(b)|+|(b \theta)| \geqslant 2\left|\left(b_{\pi(\theta)}\right)\right|
$$

where $(b \theta)=\left(b_{1} \theta_{1}, \ldots, b_{n} \theta_{n}\right)$.

P r o of. a) is obvious, since $\sum_{1}^{k} b_{i}+\sum_{n}^{l-1} b_{i}=-\sum_{k+1}^{l} b_{i}$ (due to the fact that $\sum_{1}^{n} b_{i}=0$ ) for any $l, k<l \leqslant n$.

To prove $b$ ) observe that for any $\theta$ we have by the triangle inequality $|(b)|+|(b \theta)| \geqslant 2\left|\left(b^{+}\right)\right|$and $|(b)|+|(b \theta)| \geqslant 2\left|\left(b^{-}\right)\right|$, where $\left(b^{+}\right)$is the part of $(b)$ corresponding to $\theta_{i}$ 's which are +1 's, while $\left(b^{-}\right)$corresponds to -1 's. So we can write according to a): $|(b)|+|(b \theta)| \geqslant 2\left|\left(b_{\pi(\theta)}\right)\right|$, where $\pi(\theta)$ is the permutation (dependent on $\theta$ ) so that indices of $\left(b^{+}\right)$go first, and then those of $\left(b^{-}\right)$in the reverse order.

P r o of of $\mathrm{Th}$ e or e $\mathrm{m}$ 1.1. Making use of Lemma we obtain for any collection $\left(\theta_{1}, \ldots, \theta_{n}\right)$

$$
\mathbf{E} \Phi(|(\xi)|)=\mathbf{E} \Phi(|(\xi)|+|(\xi \theta)|-|(\xi \theta)|) \geqslant \mathbf{E} \Phi\left(2\left|\left(\xi_{\pi(\theta)}\right)\right|-|(\xi \theta)|\right)
$$

(due to convexity of $\Phi$ )

$$
\geqslant 2 \mathbf{E} \Phi\left(\left|\left(\xi_{\pi(\theta)}\right)\right|\right)-\mathbf{E} \Phi(|(\xi \theta)|),
$$


where $\pi$ is a permutation of $\{1, \ldots, n\}$ dependent on the collection $\left(\theta_{1}, \ldots, \theta_{n}\right)$. Now the exchangeability of $\left(\xi_{1}, \ldots, \xi_{n}\right)$ immediately leads to a).

To prove $b$ ) we take average of (1) with respect to all collections $\left(\theta_{1}, \ldots, \theta_{n}\right)$. Then the Fubini theorem along with the Lévy inequality yields the right-hand side part of (2). The left-hand side part is the consequence of Lemma and the exchangeability of $\left(\xi_{1}, \ldots, \xi_{n}\right)$ :

$$
\begin{aligned}
\mathbf{E} \Phi\left(\left\|\sum_{1}^{n} \xi_{j} r_{j}\right\|\right) & \leqslant \mathbf{E} \Phi(|(\xi r)|) \leqslant \mathbf{E} \Phi\left(2 \max \left(\left|\left(\xi_{j}^{+}\right)\right|,\left|\left(\xi_{j}^{-}\right)\right|\right)\right. \\
& =\mathbf{E} \Phi\left(2\left|\left(\xi_{\pi(r)}\right)\right|\right)=\mathbf{E} \Phi(2|(\xi)|) .
\end{aligned}
$$

Theorem is proved.

$\mathrm{R}$ e m a r k. The condition $\sum_{1}^{n} \xi_{j}=0$ in Theorem 1.1 is imposed for simplicity of formulation. The case of general exchangeable system $\left(\xi_{1}, \ldots, \xi_{n}\right)$ can easily be reduced to the case of exchangeable system with vanishing sum by the substitution $\xi_{i}^{\prime}=\xi_{i}-\left(\sum_{1}^{n} \xi_{j}\right) / n, i=1, \ldots, n$.

Part (b) of Theorem 1.1 for the case $\Phi(t)=t^{p}, 1 \leqslant p<\infty$, has been proved in [8] (may be with a greater constant).

2. Corollaries and applications. Bounds for centered and normalized system of exchangeable random variables. Let $\left(\xi_{1}, \ldots, \xi_{n}\right)$ be a general exchangeable system of random variables with values in a Banach space $X$ (the condition $\sum_{1}^{n} \xi_{j}=0$ is not imposed). Consider the following associated exchangeable system defined by

$$
\eta_{k}=\frac{\xi_{k}-\bar{\xi}}{\left(\mathbf{E}_{r}\left\|\sum_{1}^{n}\left(\xi_{j}-\bar{\xi}\right) r_{j}\right\|^{2}\right)^{1 / 2}}, \quad k=1, \ldots, n,
$$

(we put $\eta_{k}=0$, if $\xi_{1}=\xi_{2}=\cdots=\xi_{n}$ ), where $\bar{\xi}=\left(\xi_{1}+\cdots+\xi_{n}\right) / n$, while $\mathbf{E}_{r}$ denotes the expectation with respect to the argument of $r$ 's (without loss of generality we can assume that $\xi$ 's and $r$ 's are defined on different probability spaces). Then we have $\sum_{1}^{n} \eta_{j}=0,\left(\eta_{1}, \ldots, \eta_{n}\right)$ still being an exchangeable system of $X$-valued random variables, and Theorem 1.1b) implies

Corollary 2.1. For each $p, 1 \leqslant p<\infty$, there exist absolute constants $c_{p}>0$ and $C_{p}>0$ such that for any exchangeable system $\left(\xi_{1}, \ldots, \xi_{n}\right)$ with values in a Banach space satisfying the condition

$$
\mathbf{P}\left\{\xi_{1}=\xi_{2}=\cdots=\xi_{n}\right\}=0,
$$

the following inequalities hold true

$$
c_{p} \leqslant \mathbf{E} \max _{k \leqslant n}\left\|\sum_{1}^{k} \eta_{j}\right\|^{p} \leqslant C_{p} .
$$

The right-hand side inequality is true without the restriction (3). 
P r o of. We have by Theorem 1.1b)

$$
\begin{aligned}
\mathbf{E} \max _{k \leqslant n}\left\|\sum_{1}^{k} \eta_{j}\right\|^{p} & \leqslant 2 \mathbf{E}\left\|\sum_{1}^{n} \eta_{j} r_{j}\right\|^{p} \\
& =2 \mathbf{E}_{\xi} \mathbf{E}_{r} \frac{\left\|\sum_{1}^{n}\left(\xi_{j}-\bar{\xi}\right) r_{j}\right\|^{p}}{\left(\mathbf{E}_{r}\left\|\sum_{1}^{n}\left(\xi_{j}-\bar{\xi}\right) r_{j}\right\|^{2}\right)^{p / 2}} \leqslant 2 K_{p, \mathbf{2}}^{p}
\end{aligned}
$$

where $K_{p, q}$ is Kahane's constant involved in the Kahane inequality $(1 \leqslant q$, $p<\infty)$ :

$$
\left(\mathbf{E}\left\|\sum_{1}^{n} a_{j} r_{j}\right\|^{p}\right)^{1 / p} \leqslant K_{p, q}\left(\mathbf{E}\left\|\sum_{1}^{n} a_{j} r_{j}\right\|^{q}\right)^{1 / q} .
$$

This proves the right-hand side of (4). To prove the left-hand side we note that due to condition (3) the denominator in the definition of $\eta_{k}$ does not vanish a.s. By Theorem 1.1b)

$$
\begin{aligned}
\mathbf{E} \max _{k \leqslant n}\left\|\sum_{1}^{k} \eta_{j}\right\|^{p} & \geqslant \frac{1}{2^{p}} \mathbf{E}\left\|\sum_{1}^{n} \eta_{j} r_{j}\right\|^{p} \\
& =\frac{1}{2^{p}} \mathbf{E}_{\xi} \mathbf{E}_{r} \frac{\left\|\sum_{1}^{n}\left(\xi_{j}-\bar{\xi}\right) r_{j}\right\|^{p}}{\left(\mathbf{E}_{r}\left\|\sum_{1}^{n}\left(\xi_{j}-\bar{\xi}\right) r_{j}\right\|^{2}\right)^{p / 2}} \geqslant \frac{1}{2^{p} K_{2, p}^{p}} .
\end{aligned}
$$

It is worth to point out a particular case of Corollary 2.1. Let $\xi_{1}, \ldots, \xi_{n}$ be i.i.d. real-valued random variables, and let $\zeta_{j}=\left(\xi_{j}-\bar{\xi}\right) / s$ (as before we put $\zeta_{j}=0$, if $\left.\xi_{1}=\xi_{2}=\cdots=\xi_{n}\right), j=1, \ldots, n$, where $s$ denotes the sample standard deviation, $s^{2}=\sum_{1}^{n}\left(\xi_{j}-\bar{\xi}\right)^{2} / n$.

Corollary 2.2. For any $p, 1 \leqslant p<\infty$, there exist absolute constants $d_{p}$ and $D_{p}$ such that for any i.i.d. random variables $\xi_{1}, \ldots, \xi_{n}$ with a continuous distribution

$$
d_{p} n^{p / 2} \leqslant \mathbf{E} \max _{k \leqslant n}\left(\left|\zeta_{1}+\cdots+\zeta_{k}\right|\right)^{p} \leqslant D_{p} n^{p / 2} .
$$

The right-hand side inequality is true without any assumption on the distribution of $\xi$.

Note that $d_{p}$ and $D_{p}$ (as well as $c_{p}$ and $C_{p}$ in Corollary 2.1) are not dependent on the (continuous) distribution of $\xi$.

Corollary 2.1 also implies the following Kahane type inequality between various moments.

Corollary 2.3. For any $p$ and $q, 1 \leqslant q<p<\infty$, there exists an absolute constant $M_{p, q}$ such that for any exchangeable system $\left(\xi_{1}, \ldots, \xi_{n}\right)$ of Banach space valued random variables the following inequality holds

$$
\left(\mathbf{E} \max _{k \leqslant n}\left\|\eta_{1}+\cdots+\eta_{k}\right\|^{p}\right)^{1 / p} \leqslant M_{p, q}\left(\mathbf{E} \max _{k \leqslant n}\left\|\eta_{1}+\cdots+\eta_{k}\right\|^{q}\right)^{1 / q} .
$$


Integral of $\max$ is less than max of integrals.

Corollary 2.4. If $\left(\xi_{1}, \ldots, \xi_{n}\right)$ is an exchangeable system of $X$-valued random variables with $\sum_{1}^{n} \xi_{j}=0$, then for any continuous increasing convex function $\Phi: \mathbf{R}^{+} \rightarrow \mathbf{R}^{+}, \Phi(0)=0$,

$$
\mathbf{E} \Phi\left(\max _{k \leqslant n}\left\|\sum_{1}^{k} \xi_{j}\right\|\right) \leqslant 2 \max _{k \leqslant n} \mathbf{E} \Phi\left(2\left\|\sum_{1}^{k} \xi_{j}\right\|\right) .
$$

In particular,

$$
\mathbf{E} \max _{k \leqslant n}\left\|\sum_{1}^{k} \xi_{j}\right\|^{p} \leqslant 2^{p+1} \max _{k \leqslant n} \mathbf{E}\left\|\sum_{1}^{k} \xi_{j}\right\|^{p} .
$$

P r o of. By Theorem 1.1b)

$\mathbf{E} \Phi\left(\max _{k \leqslant n}\left\|\sum_{1}^{k} \xi_{j}\right\|\right) \leqslant 2 \mathbf{E} \Phi\left(\left\|\sum_{1}^{n} \xi_{j} r_{j}\right\|\right) \leqslant 2 \mathbf{E}_{r} \mathbf{E}_{\xi} \Phi\left(\left\|\sum \xi_{j}^{+}\right\|+\left\|\sum \xi_{j}^{-}\right\|\right)$,

where $\left(\xi_{j}^{+}\right)$is subcollection of $\left(\xi_{j}\right)$ corresponding to $r_{j}=+1$, while $\left(\xi_{j}^{-}\right)$ corresponds to $r_{j}=-1$. Then we continue due to convexity of $\Phi$

$$
\leqslant \mathbf{E}_{r} \mathbf{E}_{\xi} \Phi\left(2\left\|\sum \xi_{j}^{+}\right\|\right)+\mathbf{E}_{r} \mathbf{E}_{\xi} \Phi\left(2\left\|\sum \xi_{j}^{-}\right\|\right) \leqslant 2 \max _{k \leqslant n} \mathbf{E} \Phi\left(2\left\|\sum_{1}^{k} \xi_{j}\right\|\right),
$$

and the corollary is proved.

The case of Banach lattices of some cotype. In the case $X=\mathbf{R}$ the expression $\mathbf{E}\left|\sum_{1}^{n} a_{j} r_{j}\right|^{p}$ is equivalent (Khinchine theorem) to $\left(\sum_{1}^{n} a_{i}^{2}\right)^{p / 2}$ for $1 \leqslant p<\infty$. So Theorem 1.1b) implies

Corollary 2.5. If $\left(\xi_{1}, \ldots, \xi_{n}\right)$ is an exchangeable system of real-valued random variables with $\sum \xi_{j}=0$, then for any $p, 1 \leqslant p<\infty$,

$$
c_{p} \mathbf{E}\left(\sum_{1}^{n} \xi_{j}^{2}\right)^{p / 2} \leqslant \mathbf{E} \max _{k \leqslant n}\left|\sum_{1}^{k} \xi_{j}\right|^{p} \leqslant C_{p} \mathbf{E}\left(\sum_{1}^{n} \xi_{j}^{2}\right)^{p / 2},
$$

where $c_{p}, C_{p}$ are absolute constants dependent on $p$ only.

This result can be generalized to the case of Banach lattices having some cotype $q, 2 \leqslant q<\infty$ (the $L_{p}$-spaces, $1 \leqslant p<\infty$, represent usual examples of such lattices). For such lattices there is a generalization of the Khinchine theorem due to Maurey and Pisier [8]:

$$
\mathbf{E}\left\|\sum_{1}^{n} a_{j} r_{j}\right\|^{p} \text { is equivalent to }\left\|\left(\sum_{1}^{n}\left|a_{j}\right|^{2}\right)^{1 / 2}\right\|^{p} \text {. }
$$


This fact along with Theorem $1.1 \mathrm{~b}$ ) implies

Corollary 2.6. If $\left(\xi_{1}, \ldots, \xi_{n}\right)$ is an exchangeable system of random variables with values in a Banach lattice of some cotype $q, 2 \leqslant q<\infty$, $\sum_{1}^{n} \xi_{j}=0$, then for each $p, 1 \leqslant p<\infty$,

$$
c_{p} \mathbf{E}\left\|\left(\sum_{1}^{n}\left|\xi_{j}\right|^{2}\right)^{1 / 2}\right\|^{p} \leqslant \mathbf{E} \max _{k \leqslant n}\left\|\sum_{1}^{k} \xi_{j}\right\|^{p} \leqslant C_{p} \mathbf{E}\left\|\left(\sum_{1}^{n}\left|\xi_{j}\right|^{2}\right)^{1 / 2}\right\|^{p},
$$

where $c_{p}, C_{p}$ are constants dependent on $p$ and on the Banach lattice $X$.

Spaces of type $p$. If $X$ is a space of type $p, 1 \leqslant p \leqslant 2$, then

$$
\mathbf{E}\left\|\sum_{1}^{n} a_{j} r_{j}\right\|^{p} \leqslant C \sum_{1}^{n}\left\|a_{j}\right\|^{p}
$$

where $C$ is a constant dependent on $X$. Actually this is one of equivalent definitions of a space of type $p . L_{r}$-spaces are of type $r$, if $1 \leqslant r \leqslant 2$, and of type 2 , if $r \geqslant 2$. Theorem 1.1b) for such spaces implies

Corollary $2.7([1],[8])$. If $\left(\xi_{1}, \ldots, \xi_{n}\right)$ is an exchangeable system of random variables with values in a Banach space $X$ of type $p, 1 \leqslant p \leqslant 2$, with $\sum_{1}^{n} \xi_{j}=0$, then for some constant $C>0$ dependent on the space $X$ only

$$
\mathbf{E} \max _{k \leqslant n}\left\|\sum_{1}^{k} \xi_{j}\right\|^{p} \leqslant C \mathbf{E} \sum_{1}^{n}\left\|\xi_{j}\right\|^{p}
$$

Permutations of vectors. Let $a_{1}, \ldots, a_{n}$ be a collection of elements of a normed space with $\sum_{1}^{n} a_{j}=0$. In a series of problems of analysis of interest is the estimation of

$$
\operatorname{Ave}_{\pi} \Phi\left(\max _{k \leqslant n}\left\|\sum_{1}^{k} a_{\pi(j)}\right\|\right)=\frac{1}{n !} \sum_{\pi} \Phi\left(\max _{k \leqslant n}\left\|\sum_{1}^{k} a_{\pi(j)}\right\|\right),
$$

where $\pi$ is a permutation of $\{1, \ldots, n\}$, while the summation is carried out over all such permutations. Theorem 1.1 permits to find a proper bilateral estimation for this quantity.

Corollary 2.8. Let $\Phi: \mathbf{R}^{+} \rightarrow \mathbf{R}^{+}$be a continuous increasing convex function, $\Phi(0)=0$.

a) The following bilateral inequality holds true

$$
\mathbf{E} \Phi\left(\frac{1}{2}\left\|\sum_{1}^{n} a_{j} r_{j}\right\|\right) \leqslant \operatorname{Ave}_{\pi} \max _{k \leqslant n} \Phi\left(\left\|\sum_{1}^{k} a_{\pi(j)}\right\|\right) \leqslant 2 \mathbf{E \Phi}\left(\left\|\sum_{1}^{n} a_{j} r_{j}\right\|\right) .
$$

b) If $X=\mathbf{R}$ and $1 \leqslant p<\infty$, then

$$
\frac{1}{2^{p} c_{p}^{p}}\left(\sum_{1}^{n} a_{j}^{2}\right)^{p / 2} \leqslant \mathrm{Ave}_{\pi} \max _{k \leqslant n}\left|\sum_{1}^{k} a_{\pi(j)}\right|^{p} \leqslant 2 c_{p}^{p}\left(\sum_{1}^{n} a_{j}^{2}\right)^{p / 2},
$$


where $c_{p}$ is the Khinchine constant taking part in the inequality

$$
\mathbf{E}\left|\sum a_{j} r_{j}\right|^{p} \leqslant c_{p}^{p}\left(\sum_{1}^{n} a_{j}^{2}\right)^{p / 2} .
$$

P r o of. Consider the random variables $\xi_{1}, \ldots, \xi_{n}$ associated with $a_{1}, \ldots, a_{n}$. They are defined on the space $\Pi$ of all permutations of $\{1, \ldots, n\}$ with the uniform distribution as follows: $\xi_{i}(\pi)=a_{\pi(i)}, \pi \in \Pi, i=1, \ldots, n$. It is easy to see that the system $\left(\xi_{1}, \ldots, \xi_{n}\right)$ is exchangeable and $\sum_{1}^{n} \xi_{i}=0$. Now application of Theorem 1.1 implies Corollary 2.8 .

The inequality in b) of Corollary 2.8 (the most important right-hand side of it) has been established by Garsia [4] in connection with proving a.e. convergence of rearranged orthogonal series. The vector case for the power function $\Phi(t)=t^{p}, 1 \leqslant p<\infty$, has been treated by Maurey and Pisier [8].

Corollary 2.8a) implies the following result playing a crucial role in problems of rearrangements of functional series.

Proposition. Assume that for a series $\sum_{1}^{\infty} a_{j}$ in a normed space $X$, a subsequence $S_{n_{k}}$ of the sequence of partial sums converges to an element $s \in X$. Then if $\sum_{1}^{\infty} a_{j} r_{j}$ converges a.s. in $X$, then there exists a permutation $\pi: \mathbf{N} \rightarrow \mathbf{N}$ such that the series $\sum_{1}^{\infty} a_{\pi(j)}$ converges to $s$.

The reader is referred to [2] and [3] for a refinement of the last proposition and its applications.

3. Inequalities for tails. Moment inequalities established in Section 1 prompt that there should be a relation between tail probabilities of $\max _{k \leqslant n}\left\|\xi_{1}+\cdots+\xi_{k}\right\|$ and $\left\|\sum_{1}^{n} \xi_{j} r_{j}\right\|$. In the next statement we establish such a relation.

Theorem 3.1. Let $\left(\xi_{1}, \ldots, \xi_{n}\right)$ be an exchangeable system of random variables with $\sum_{1}^{n} \xi_{i}=0$ defined on $(\Omega, \mathscr{A}, \mathbf{P})$ and taking values in a Banach space $X$. Then for any $t>0$ the following inequalities hold true

$$
\begin{aligned}
(\lambda \times \mathbf{P})\left\{\left\|\sum_{1}^{n} \xi_{j} r_{j}\right\|>2 t\right\} & \leqslant \mathbf{P}\left\{\max _{k \leqslant n}\left\|\xi_{1}+\cdots+\xi_{k}\right\|>t\right\} \\
& \leqslant 10(\lambda \times \mathbf{P})\left\{\left\|\sum_{1}^{n} \xi_{j} r_{j}\right\|>\frac{t}{4}\right\}
\end{aligned}
$$

where $r_{1}, \ldots, r_{n}$ are Rademacher functions defined on $[0,1]$ with the Lebesgue measure $\lambda$.

We reduce Theorem 3.1 to the following assertion proved in [2]. For the one-dimensional version see also [6]. 
Theorem 3.1'. Let $\left(a_{1}, \ldots, a_{n}\right)$ be any collection of elements of $a$ normed space $X$ with $\sum_{1}^{n} a_{j}=0$. Then the following bilateral inequality holds

$$
\begin{aligned}
\lambda\left\{\left\|\sum_{1}^{n} a_{j} r_{j}\right\|>2 t\right\} & \leqslant \frac{1}{n !} \operatorname{card}\left\{\pi: \max _{k \leqslant n}\left\|a_{\pi(1)}+\cdots+a_{\pi(k)}\right\|>t\right\} \\
& \leqslant 10 \lambda\left\{\left\|\sum_{1}^{n} a_{j} r_{j}\right\|>\frac{t}{4}\right\} .
\end{aligned}
$$

P r o of of $\mathrm{Th}$ e o r e m 3.1. Using the exchangeability of the system $\left(\xi_{1}, \ldots, \xi_{n}\right)$ we can write for any permutation $\pi:\{1, \ldots, n\} \rightarrow\{1, \ldots, n\}$

$$
\mathbf{P}\left\{\max _{k \leqslant n}\left\|\xi_{1}+\cdots+\xi_{k}\right\|>t\right\}=\mathbf{P}\left\{\max _{k \leqslant n}\left\|\xi_{\pi(1)}+\cdots+\xi_{\pi(k)}\right\|>t\right\} .
$$

Integrating both sides with respect to the uniform distribution $m$ on the set $\Pi$ of all permutations we get

$$
\mathbf{P}\left\{\max _{k \leqslant n}\left\|\xi_{1}+\cdots+\xi_{k}\right\|>t\right\}=\int_{\Pi} \mathbf{P}\left\{\max _{k \leqslant n}\left\|\xi_{\pi(1)}+\cdots+\xi_{\pi(k)}\right\|>t\right\} d m(\pi)
$$

(by the Fubini theorem)

$$
=\int_{\Omega} m\left\{\max _{k \leqslant n}\left\|\xi_{\pi(1)}+\cdots+\xi_{\pi(k)}\right\|>t\right\} d \mathbf{P}\{\omega\}
$$

(by Theorem 3.1')

$$
\leqslant 10 \int_{\Omega} \lambda\left\{\left\|\sum_{1}^{n} \xi_{j} r_{j}\right\|>\frac{t}{4}\right\} d \mathbf{P}=10(\lambda \times \mathbf{P})\left\{\left\|\sum_{1}^{n} \xi_{j} r_{j}\right\|>\frac{t}{4}\right\} .
$$

This proves the right-hand side inequality. The left-hand side part can be treated in the similar way.

Corollaries. Note first that to any system of random variables $\left(\xi_{1}, \ldots, \xi_{n}\right)$ one can associate an exchangeable system on some extension of the underlying probability space $(\Omega, \mathscr{A}, \mathbf{P})$. Namely, it is easy to see that the system of random variables $\xi_{k}(\omega, \pi)=\xi_{\pi(k)}(\omega),(\omega, \pi) \in \Omega \times \Pi, k=1, \ldots, n$, where $\Pi$ as before is the set of all permutations of $\{1, \ldots, n\}$, is an exchangeable system. Applying Theorem 3.1 to the system we come to the following assertion.

Corollary 3.1. Let $\left(\xi_{1}, \ldots, \xi_{n}\right)$ be any collection of random variables with values in a Banach space $X$, and let $\left(\eta_{1}, \ldots, \eta_{n}\right)$ be the associated collection of random variables (i.e., centered and normalized as in Section 2). Then for any $t>0$ the following bilateral inequality holds

$$
\begin{aligned}
(\lambda \times \mathbf{P})\left\{\left\|\sum_{1}^{n} \eta_{j} r_{j}\right\|>2 t\right\} & \leqslant \frac{1}{n !} \sum_{\pi} \mathbf{P}\left\{\max _{k \leqslant n}\left\|\eta_{\pi(1)}+\cdots+\eta_{\pi(k)}\right\|>t\right\} \\
& \leqslant 10(\lambda \times \mathbf{P})\left\{\left\|\sum_{1}^{n} \eta_{j} r_{j}\right\|>\frac{t}{4}\right\} .
\end{aligned}
$$


Moment maximal inequality for non-convex functions. Theorem 3.1 allows one to extend Theorem $1.1 \mathrm{~b}$ ) to the case of non-convex functions $\Phi: \mathbf{R}^{+} \rightarrow \mathbf{R}^{+}$.

Corollary 3.2. Let $\left(\xi_{1}, \ldots, \xi_{n}\right)$ be a system of exchangeable Banach space valued random variables with $\sum_{1}^{n} \xi_{j}=0$, and let $\Phi: \mathbf{R}^{+} \rightarrow \mathbf{R}^{+}, \Phi(0)=$ 0 , be any continuous increasing (not necessarily convex) function. Then the following bilateral inequality holds:

$$
\mathbf{E} \Phi\left(\frac{1}{2}\left\|\sum_{1}^{n} \xi_{i} r_{i}\right\|\right) \leqslant \mathbf{E} \Phi\left(\max _{1 \leqslant k \leqslant n}\left\|\xi_{1}+\cdots+\xi_{k}\right\|\right) \leqslant 10 \mathbf{E} \Phi\left(4\left\|\sum_{1}^{n} \xi_{j} r_{j}\right\|\right) \text {. }
$$

$\mathrm{Pr}$ o of. The integration by parts formula yields

$$
\mathbf{E} \boldsymbol{\Phi}\left(\max _{1 \leqslant k \leqslant n}\left\|\xi_{1}+\cdots+\xi_{k}\right\|\right)=\int_{0}^{\infty} \mathbf{P}\left\{\max _{1 \leqslant k \leqslant n}\left\|\xi_{1}+\cdots+\xi_{k}\right\|>t\right\} d \Phi(t)
$$

(due to Theorem 3.1)

$$
\leqslant 10 \int_{0}^{\infty} \mathbf{P}\left\{\left\|\sum_{1}^{n} \xi_{k} r_{k}\right\|>\frac{t}{4}\right\} d \Phi(t)=\mathbf{E} \Phi\left(4\left\|\sum_{1}^{n} \xi_{k} r_{k}\right\|\right) .
$$

This proves the right-hand side inequality. The proof of the left-hand side part is similar.

4. Inequalities with multipliers. Our next goal is to estimate $\mathbf{E} \max _{k \leqslant n}\left\|\sum_{1}^{n} \mu_{j} \xi_{j}\right\|$ with numerical multipliers $\mu_{1}, \ldots, \mu_{n}$. Although our inequalities are no longer bilateral, in the particular case of $\mu_{1}=\mu_{2}=\cdots=$ $\mu_{n}$ they give (up to a constant factor) the right-hand side inequality of Theorem 1.1. We follow mainly the method of reducing to the case of equal coefficients suggested by Maurey and Pisier [8]. As we will see the method permits to treat the case when $\mu_{i}$ 's are operators.

Theorem 4.1. Let $\left(\xi_{1}, \ldots, \xi_{n}\right)$ be an exchangeable system of random variables with values in a Banach space $X, \sum_{1}^{n} \xi_{j}=0$. Then for any bounded linear operators $A_{1}, \ldots, A_{n}$ in $X$ and any $p, 1<p<\infty$, the following inequality holds

$$
\begin{aligned}
\left(\mathbf{E} \max _{k \leqslant n}\left\|\sum_{1}^{k} A_{j} \xi_{j}\right\|^{p}\right)^{1 / p} \leqslant & \frac{6 p^{2}}{(p-1)^{2}}\left(\mathbf{E}\left\|\sum_{1}^{n} A_{j} \xi_{j} r_{j}\right\|^{p}\right)^{1 / p} \\
& +\frac{8(4 p-1)}{(9 p-1)(n-1)}\left(\mathbf{E}\left\|\sum_{1}^{n} \xi_{j} r_{j}\right\|^{p}\right)^{1 / p} \sum_{1}^{n}\left\|A_{j}\right\|,
\end{aligned}
$$

where $\left(r_{j}\right)$ is a family of Rademacher random variables which is independent of $\left(\xi_{1}, \ldots, \xi_{n}\right)$.

P r o o f. Denote

$$
\varphi_{k}^{*}\left(A_{1}, \ldots, A_{k}\right)=\left(\mathbf{E} \max _{j \leqslant k}\left\|\sum_{1}^{j} A_{i} \xi_{i}\right\|^{p}\right)^{1 / p},
$$




$$
\varphi_{k}\left(A_{1}, \ldots, A_{k}\right)=\left(\mathbf{E}\left\|\sum_{1}^{k} A_{j} \xi_{j}\right\|^{p}\right)^{1 / p} .
$$

It is easy to see that the exchangeability of $\left(\xi_{1}, \ldots, \xi_{n}\right)$ implies that for any $i<n$

$$
\mathbf{E}\left(\xi_{j_{1}} \mid \xi_{1}, \ldots, \xi_{i}\right)=\mathbf{E}\left(\xi_{j_{2}} \mid \xi_{1}, \ldots, \xi_{i}\right) \text { for any } j_{1}, j_{2}>i \text {. }
$$

Therefore we have for any $i \leqslant k$ (taking into account that $\sum_{1}^{n} \xi_{j}=0$ )

$$
\begin{aligned}
\mathbf{E}\left(\sum_{j=1}^{k} A_{j} \xi_{j} \mid \xi_{1}, \ldots, \xi_{i}\right) & =\sum_{1}^{i} A_{j} \xi_{j}+\mathbf{E}\left(\sum_{i+1}^{k} A_{j} \xi_{j} \mid \xi_{1}, \ldots, \xi_{i}\right) \\
& =\sum_{1}^{i} A_{j} \xi_{j}+\sum_{i+1}^{k} A_{j} \frac{1}{n-i} \mathbf{E}\left(\sum_{l=i+1}^{n} \xi_{l} \mid \xi_{1}, \ldots, \xi_{i}\right) \\
& =\sum_{1}^{i} A_{j} \xi_{j}-\frac{1}{n-i}\left(\sum_{i+1}^{k} A_{j}\right)\left(\sum_{1}^{i} \xi_{j}\right) .
\end{aligned}
$$

Since $\mathbf{E}\left(\sum_{j=1}^{k} A_{j} \xi_{j} \mid \xi_{1}, \ldots, \xi_{i}\right)$ is a martingale with respect to $i$, we have according to the Doob maximal inequality

$$
\varphi_{k}^{*}\left(A_{1}, \ldots, A_{k}\right) \leqslant q \varphi_{k}\left(A_{1}, \ldots, A_{k}\right)+\frac{1}{n-k} \sum_{1}^{k}\left\|A_{j}\right\| \varphi_{k}^{*}(I, \ldots, I),
$$

where $q=p /(p-1)$, while $I$ is the identity operator in $X$.

Let now $\theta_{1}, \ldots, \theta_{k}$ be an arbitrary collection of signs, and let $\sigma$ be a permutation of $\{1, \ldots, k\}$ such that in the ordered collection $\left(\theta_{\sigma(1)}, \ldots, \theta_{\sigma(k)}\right)$ beforehand there are pluses in a row followed by minuses. The triangle inequality argument allows one to write $\varphi_{k}\left(A_{\sigma(1)}, \ldots, A_{\sigma(k)}\right) \leqslant$ $\varphi_{k}^{*}\left(A_{\sigma(1)}, \ldots, A_{\sigma(k)}\right) \leqslant 3 \varphi_{k}^{*}\left(\theta_{\sigma(1)} A_{\sigma(1)}, \ldots, \theta_{\sigma(k)} A_{\sigma(k)}\right)$. Using (5) together with the fact that the family $\left(\xi_{j}\right)$ is exchangeable we get from here

$$
\begin{aligned}
\varphi_{k}\left(A_{1}, \ldots, A_{k}\right)= & \varphi_{k}\left(A_{\sigma(1)}, \ldots, A_{\sigma(k)}\right) \leqslant 3 q \varphi_{k}\left(\theta_{\sigma(1)} A_{\sigma(1)}, \ldots, \theta_{\sigma(k)} A_{\sigma(k)}\right) \\
& +\frac{3}{n-k}\left(\sum_{1}^{k}\left\|A_{j}\right\|\right) \varphi_{k}^{*}(I, \ldots, I) .
\end{aligned}
$$

Substituting this expression for $\varphi_{k}$ in (5) we have

$$
\begin{aligned}
\varphi_{k}^{*}\left(A_{1}, \ldots, A_{k}\right) \leqslant & 3 q^{2} \varphi_{k}\left(\theta_{1} A_{1}, \ldots, \theta_{k} A_{k}\right) \\
& +\frac{3 q+1}{n-k} \sum_{1}^{k}\left\|A_{j}\right\| \varphi_{k}^{*}(I, \ldots, I) .
\end{aligned}
$$

Analogously we can estimate the quantity $\psi_{n-k}^{*}\left(A_{k+1}, \ldots, A_{n}\right)=$ $\left(\mathbf{E} \max _{k+1 \leqslant i \leqslant n}\left\|\sum_{k+1}^{i} A_{j} \xi_{j}\right\|^{p}\right)^{1 / p}$ :

$$
\begin{aligned}
\psi_{n-k}^{*}\left(A_{k+1}, \ldots, A_{n}\right) \leqslant & 3 q^{2} \psi_{n-k}\left(\theta_{k+1} A_{k+1}, \ldots, \theta_{n} A_{n}\right) \\
& +\frac{3 q+1}{k} \sum_{k+1}^{n}\left\|A_{j}\right\| \psi_{n-k}^{*}(I, \ldots, I),
\end{aligned}
$$


where $\psi_{n-k}\left(A_{k+1}, \ldots, A_{n}\right)=\left(\mathbf{E}\left\|\sum_{k+1}^{n} A_{j} \xi_{j}\right\|^{p}\right)^{1 / p}$. Now put $k=[n / 2]$. By averaging both sides of $(6)$ and (7) with respect to $\theta=\left(\theta_{1}, \ldots, \theta_{k}\right)$ we obtain by Theorem 1.1

$$
\begin{aligned}
\varphi_{n}^{*}\left(A_{1}, \ldots, A_{n}\right) \leqslant & \varphi_{k}^{*}\left(A_{1}, \ldots, A_{k}\right)+\psi_{n-k}^{*}\left(A_{k+1}, \ldots, A_{n}\right) \\
\leqslant & 6 q^{2}\left(\mathbf{E}\left\|\sum_{1}^{n} A_{j} \xi_{j} r_{j}\right\|^{p}\right)^{1 / p} \\
& +\frac{8(3 q+1)}{n-1} \sum_{1}^{n}\left\|A_{j}\right\|\left(\mathbf{E}\left\|\sum_{1}^{n} \xi_{j} r_{j}\right\|^{p}\right)^{1 / p},
\end{aligned}
$$

where $\left(r_{j}\right)$ is a Rademacher system of random variables which is independent of $\left(\xi_{i}\right)$. Theorem 4.1 is proved.

Theorem 4.1 implies the following

Corollary 4.1. Let $\left(\xi_{1}, \ldots, \xi_{n}\right)$ be an exchangeable system of $X$-valued random variables, $\sum_{1}^{n} \xi_{i}=0$. Then

a) If $X$ is of type $p, 1<p \leqslant 2$, then there exists a constant $c_{p}(X)$ such that for any bounded linear operators $A_{1}, \ldots, A_{n}$ in $X$

$$
\mathbf{E} \max _{k \leqslant n}\left\|\sum_{1}^{k} A_{j} \xi_{j}\right\|^{p} \leqslant c_{p}(X) \mathbf{E}\left\|\xi_{1}\right\|^{p} \sum_{1}^{n}\left\|A_{j}\right\|^{p} ;
$$

b) If $X=\mathbf{R}$, while $A_{1}, \ldots, A_{n}$ are reals, then

$$
\mathbf{E} \max _{k \leqslant n}\left|\sum_{1}^{n} A_{j} \xi_{j}\right|^{2} \leqslant 18496 \mathbf{E} \xi_{1}^{2} \sum_{1}^{n} A_{i}^{2} .
$$

Let us give one more consequence of Theorem 4.1.

Corollary 4.2. If $\left(\xi_{1}, \ldots, \xi_{n}\right)$ is an exchangeable system of orthonormal random variables, then for any collection of reals $\alpha_{1}, \ldots, \alpha_{n}$ the following inequality holds

$$
\mathbf{E} \max _{k \leqslant n}\left|\sum_{1}^{k} \alpha_{j} \xi_{j}\right|^{2} \leqslant c \sum_{1}^{n} \alpha_{j}^{2},
$$

where $c$ is an absolute constant ( $c \leqslant 18497)$.

Corollary 4.2 has been proved by Garsia [4] to show that any orthonormal exchangeable system $\left(\xi_{j}\right)_{1}^{\infty}$ is a system of convergence: any series $\sum_{1}^{\infty} \alpha_{j} \xi_{j}$ with $\sum_{1}^{\infty} \alpha_{j}^{2}<\infty$ is a.s. convergent.

Finally we give a result estimating

$$
\frac{1}{n !} \sum_{\pi} \max _{k \leqslant n}\left\|\mu_{1} a_{\pi(1)}+\cdots+\mu_{k} a_{\pi(k)}\right\|^{2},
$$

where as before $a_{1}, \ldots, a_{n}$ are elements of a normed space $X, \pi$ runs over the set $\Pi$ of all permutations of $\{1, \ldots, n\}$, while $\mu_{1}, \ldots, \mu_{n}$ are real numbers with the fixed positions in the sum. As before we consider the exchangeable 
system of generated $X$-valued random variables $\xi_{j}(\pi) a_{\pi(j)}, j=1, \ldots, n$, $\pi \in \Pi$, and apply Corollary 4.1 to obtain the following assertion.

Corollary 4.3. If $a_{1}, \ldots, a_{n}$ are elements of a Banach space of type 2 , while $\mu_{1}, \ldots, \mu_{n}$ are real numbers, then

$$
\frac{1}{n !} \sum_{\pi} \max _{k \leqslant n}\left\|\mu_{1} a_{\pi(1)}+\cdots+\mu_{k} a_{\pi(k)}\right\|^{2} \leqslant \frac{C \sum_{1}^{n} \mu_{k}^{2}}{n}\left(\sum_{1}^{n}\left\|a_{j}\right\|^{2}+\left\|\sum_{1}^{n} a_{j}\right\|^{2}\right) \text {, }
$$

where $C$ is a constant dependent on the space $X$ only.

The proof can easily be derived from Corollary 4.1 first for the case when $\sum_{1}^{n} a_{i}=0$ and then for the general case by use of the Hölder inequality.

The Hilbert space case of Corollary 4.3 has been used in [3] to establish the permutational Kolmogorov SLLN for orthogonal random variables.

In the case $X=\mathbf{R}$ Corollary 4.3 has been established first in [4].

Acknowledgment. The authors express their gratitude to Professor Shlomo Levental for valuable discussions.

\section{REFERENCES}

1. Assouad P. Espaces $p$-lisses, rearrangements. - Seminaire Maurey-Schwartz 197475, exp. XVI, 1975, $7 \mathrm{p}$.

2. Chobanyan $S$. Convergence a.s. of rearranged random series in Banach space and associated inequalities. - Probability in Banach Spaces. V. 9. Ed. by J. HoffmanJørgensen et al. Boston: Birkhäuser, 1994, p. 3-29.

3. Chobanyan S., Mandrekar V. Almost everywhere convergence and SLLN under rearrangement. - Stochastic Processes and Related Topics. In memory of Stamatis Cambanis 1943-1995. Ed. by I. Karatzas et al. Boston: Birkhäuser, p. 25-34.

4. Garsia A. Topics in Almost Everywhere Convergence. Chicago: Markham, 1970.

5. Kadets V.M., Kadets M. I. Rearrangements of Series in Banach Spaces. Providence, RI: Amer. Math. Soc., 1991.

6. Кашия Б. С., Саакяя А. А. Ортогональные ряды. М.: Наука, 1984.

7. Latata $R$., Oleszkiewicz K. On the best constant in the Khinchine-Kahane inequality. - Studia Math., 1994, v. 109, p. 101-104.

8. Maurey B., Pisier G. Remarques sur l'exposé d'Assouad. - Seminaire MaureySchwartz 1974-75, Annexe 1, 1975.

9. Нихишия E.M. О сходяшихся перестановках функциональных рядов. - Матем. заметки, 1967, т. 1, с. 129-136. 